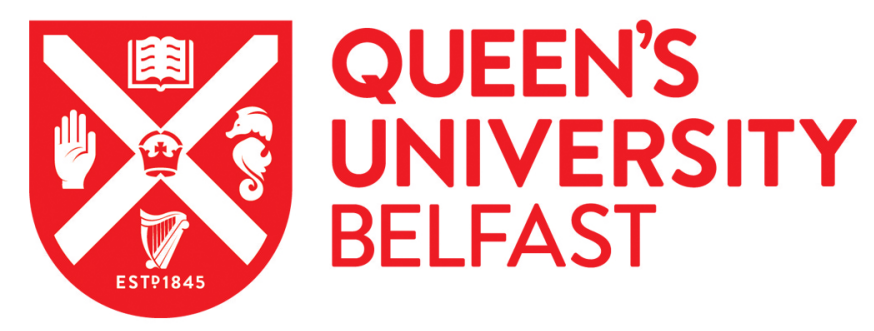

\title{
Assisted Diagnosis of Cervical Intraepithelial Neoplasia (CIN)
}

Wang, Y., Crookes, D., Eldin, O. S., Wang, S., Hamilton, P., \& Diamond, J. (2009). Assisted Diagnosis of Cervical Intraepithelial Neoplasia (CIN). IEEE Journal of Selected Topics in Signal Processing, Special Issue on Digital Image Processing Techniques for Oncology, 3(1), 112-121. https://doi.org/10.1109/JSTSP.2008.2011157

\section{Published in:}

IEEE Journal of Selected Topics in Signal Processing, Special Issue on Digital Image Processing Techniques for Oncology

\section{Queen's University Belfast - Research Portal:}

Link to publication record in Queen's University Belfast Research Portal

\section{General rights}

Copyright for the publications made accessible via the Queen's University Belfast Research Portal is retained by the author(s) and / or other copyright owners and it is a condition of accessing these publications that users recognise and abide by the legal requirements associated with these rights.

Take down policy

The Research Portal is Queen's institutional repository that provides access to Queen's research output. Every effort has been made to ensure that content in the Research Portal does not infringe any person's rights, or applicable UK laws. If you discover content in the Research Portal that you believe breaches copyright or violates any law, please contact openaccess@qub.ac.uk. 


\title{
Assisted Diagnosis of Cervical Intraepithelial Neoplasia (CIN)
}

\author{
Yinhai Wang, Danny Crookes, Member, IEEE, Osama Sharaf Eldin, Shilan Wang, Peter Hamilton, and \\ Jim Diamond
}

\begin{abstract}
This paper introduces an automated computerassisted system for the diagnosis of cervical intraepithelial neoplasia (CIN) using ultra-large cervical histological digital slides. The system contains two parts: the segmentation of squamous epithelium and the diagnosis of CIN. For the segmentation, to reduce processing time, a multiresolution method is developed. The squamous epithelium layer is first segmented at a low (2X) resolution. The boundaries are further fine tuned at a higher (20X) resolution. The block-based segmentation method uses robust texture feature vectors in combination with support vector machines (SVMs) to perform classification. Medical rules are finally applied. In testing, segmentation using 31 digital slides achieves $94.25 \%$ accuracy. For the diagnosis of CIN, changes in nuclei structure and morphology along lines perpendicular to the main axis of the squamous epithelium are quantified and classified. Using multi-category SVM, perpendicular lines are classified into Normal, CIN I, CIN II, and CIN III. The robustness of the system in term of regional diagnosis is measured against pathologists' diagnoses and inter-observer variability between two pathologists is considered. Initial results suggest that the system has potential as a tool both to assist in pathologists' diagnoses, and in training.
\end{abstract}

Index Terms-Cervical cancer, CIN, diagnosis, digital pathology, digital slide, image processing, SVM.

\section{INTRODUCTION}

$\mathbf{C}$ ERVICAL cancer is the second most common cancer in women worldwide, and it is the principal cancer of women in most developing countries, where 80 percent of cases occur [1]. Cervical intraepithelial neoplasia (CIN) is a pre-malignant condition where abnormal cells are restricted to the epithelial layer. CIN demonstrates progressive stages with increasing numbers of abnormal cells and can lead to cancer once the neoplastic cells extend beyond the epithelium basement membrane, invade adjacent tissues and spread to other tissues and organs. The diagnosis and grading of CIN is based on the interpretation of visually assessed features of cervical histological slides. Diagnostic decisions are generally

Manuscript received April 15, 2008; revised October 15, 2008. Current version published February 19, 2009. The associate editor coordinating the review of this manuscript and approving it for publication was Dr. Jianhua Yao.

Y. Wang and D. Crookes are with the School of Electronics, Electrical Engineering, and Computer Science, Queen's University Belfast, Belfast BT3 9DT U.K. (e-mail: y.wang@qub.ac.uk).

O. S. Eldin is with the School of Medicine and Medical Science, University College Dublin, Dublin 4, Ireland.

S. Wang is with the Shanxi Modern Women Hospital, Bosheng Medical Investment Co., Ltd., Taiyuan, Shanxi 030012, China.

P. Hamilton and J. Diamond are with the Centre for Cancer Research and Cell Biology, Queen's University Belfast, Belfast BT9 7BL U.K.

Color versions of one or more of the figures in this paper are available online at http://ieeexplore.ieee.org.

Digital Object Identifier 10.1109/JSTSP.2008.2011157

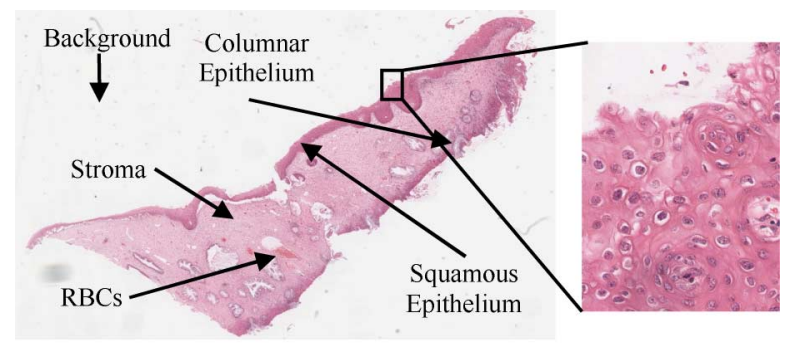

(a)

(b)

Fig. 1. Example of a digital slide. (a) Digital slide of $53450 \times 38090$ pixels at $40 \mathrm{X}$ magnification. (b) Zoomed-in view of the region in square.

made by a pathologist. This process can be extremely subjective, resulting in inter- and intra-observer variation and poor reproducibility in the grading of cervical lesions [2]-[4].

Digital pathology is the management and interpretation of pathology information from virtual, digital microscopy [5]. Compared to traditional pathology, a major advantage of digital pathology is that slides can be analysed using software rather than limited manual analysis [5], [6]. A digital slide is a high-resolution scan of a microscope tissue sample. A typical cervical histological slide, which has a tissue area of $30 \mathrm{~mm}$ $\times 20 \mathrm{~mm}$, is scanned to give an image of $120000 \times 80000$ pixels, or $28 \mathrm{~GB}$ [7] of uncompressed color image data. An example of a digital slide can be seen in Fig. 1.

In recent research in digital pathology, studies [8]-[10] investigate the qualities of scanned digital slides compared with traditional glass slides. Other studies are on viewing digital slides, such as for education [11]-[13] and telepathology [14], [15]. The analysis of digital slides is still at a preliminary stage, with investigations on tissue microarrays (TMAs) [16]-[18] as well as the analysis of whole slides [19], [20].

In this study, we present one of the first major investigations into the analysis of digital slides by proposing an automated system for the diagnosis of CIN using digital slides. The proposed system contains two phases: the segmentation of squamous epithelium and the diagnosis of CIN.

\section{Methodology}

Glass slides were scanned at 40X objective magnification (a resolution of $0.25 \mu \mathrm{m} /$ pixel) using an Aperio ScanScope CS scanner and archived in 24-bit color JPEG format. Based on 31 available digital slides, the sizes of scanned slides vary and are up to $31.4 \mathrm{~GB}$ of uncompressed data, which reflects a region of 
$16.65 \mathrm{~mm} \times 35.70 \mathrm{~mm}$ of a glass slide. All 31 slides were diagnosed, region by region, by one pathologist. 20 of them were diagnosed by two pathologists independently.

For the segmentation of squamous epithelium, a region-based multi-resolution texture classification method is used. Given the large nature of digital slides, it is very time consuming to do segmentation at the maximum resolution (40X magnification). Our strategy is therefore to do an initial block-based coarse segmentation at a low resolution, and then fine tune the boundary blocks at a higher resolution.

This immediately raises two issues to be investigated: what is the best resolution and block size, both for coarse segmentation and for boundary tuning. These parameters are important for reducing processing time, while retaining the quality of segmentation. These questions are answered in Section III-E.

The presence of CIN in a squamous epithelium region can be determined by exploring the quantitative changes that occur progressively along lines perpendicular to the medial axis of the squamous epithelium. Feature vectors for pixel blocks along each perpendicular line are obtained from the measurements of nuclei patterns along each line. The feature vectors for each line are classified using multicategory SVM. Combining perpendicular line classification results within each region gives a regional diagnosis of CIN for a digital slide.

\section{Segmentation of Squamous Epithelium}

At first sight, it might appear that a simple segmentation approach based on color and morphology might be sufficient. However, such is the range and variability of these features across different batches (and even within the same image), a more sophisticated and robust approach proved necessary.

A test image dataset is used to identify texture features in conjunction with SVM for segmentation. We then present an 8 -stage multi-resolution segmentation process and demonstrate its performance with a set of test results.

\section{A. Test Image Dataset for Segmentation}

A first batch of $20 \mathrm{H} \& \mathrm{E}$ stained cervical histological slides were selected for the training and testing. These slides reflect varying diagnostic outcomes, including Normal, CIN I, CIN II, and CIN III. Under supervision of a pathologist, each slide was examined and annotated to be five major different regions: squamous epithelium, stroma, background, columnar epithelium and red blood cells (RBCs). These annotated slides were used as the "ground truth" for training and testing.

\section{B. Image Block Database}

To segment a complete digital slide, we divide the image into a large set of blocks, and classify each block into one of the above five categories. Each block needs to be large enough to demonstrate the characteristics of a particular region type (e.g., stroma). However, since a block is normally the lowest resolution of segmentation, a block must be small enough to give sufficient accuracy, particularly along boundaries. For this reason, image blocks were initially chosen to be $500 \times 500$ pixels at 40X magnification. A selection of representative blocks from the 20 slides was made for each category, which resulted in 1618 blocks per category (this figure was based on the incidence of the rarer categories in our set of digital slides).

\section{Texture Features}

A range of possible texture features was initially identified based on the literature and on their use in similar research areas. Based on subsequent tests, the most useful features were selected to form feature vectors.

Texture features can be measured using statistical, structural and spectral approaches [21]. The statistical approach, which uses statistical moments and gray level co-occurrence matrix (GLCM), is perhaps the most widely used. Therefore, texture features from this category were investigated and selected.

Statistical moments estimate individual pixel properties, such as used in [22], [23]. We select six statistical moments-average intensity $(m)$, average contrast $(\sigma)$, smoothness $(R)$, third moment $\left(\mu_{3}\right)$, uniformity $(U)$ and entropy $(e)$. GLCM related textures estimate properties of two or more pixel values occurring at specific locations relative to each other. They have been used for various classification tasks [24]-[29]. Studies [24], [27], [28] suggest GLCM features can be used to distinguish different tissue regions, especially contrast $\left(F_{C o n}^{\mathrm{Har}}\right)$, correlation $\left(F_{\mathrm{Cor}}^{\mathrm{Har}}\right)$, angular second moment $\left(F_{A S M}^{H a r}\right)$, and inverse difference moment $\left(F_{I D M}^{H a r}\right)$. Therefore, they are also selected. For the calculation of GLCM, the immediate neighbourhood pixels within a distance of 1 in both vertical and horizontal directions are selected. This gives eight GLCM related textures.

\section{Support Vector Machine}

Support Vector Machine was initially introduced in [30] and has been developed further since then. It is a robust learning machine for two or more category classification problems. For classification into two categories, SVM solves the problem of obtaining the optimal boundary hyperplane which separates the feature vectors of the two categories. The basic idea of SVM is to locate the boundary which is most distant from the nearest vectors of both of the two categories.

To classify into our five categories using SVM, a hierarchy of four binary classifications is identified. These four binary SVM classifications use different sets of texture features. The optimal set of features for each classifier is selected by systematic testing on the test data set. The differentiation between background and tissue component only needs eight features, namely $m, \sigma, R$, $U, e, F_{C o n}^{\mathrm{Har}}, F_{A S M}^{\mathrm{Har}}$ and $F_{I D M}^{\mathrm{Har}}\left(F_{C o n}^{\mathrm{Har}}, F_{A S M}^{\mathrm{Har}}\right.$ and $F_{I D M}^{\mathrm{Har}}$ are calculated with their GLCM offsets in the horizontal direction only). The other three binary classifications need all 14 texture features.

Binary classification of background and tissue is performed first. Then tissue is subclassified as either squamous epithelium or stroma. The resulting squamous epithelium may contain some columnar epithelium and/or RBCs, and so two final classifications identify columnar epithelium and RBCs.

The popular LibSVM [31] tool is used to perform SVM classifications, with the widely used Gaussian RBF kernel. There are two parameters to be decided to get optimised support vectors: the penalty parameter $C$ from the soft margin method, and $\gamma$ from the Gaussian RBF kernel. N-fold cross-validation and grid-search is performed to get the best combination of $C$ and $\gamma$. 
TABLE I

GRID-SEARCH FOR BLOCK SIZE AND RESOLUTION FOR THE CLASSIFICATION BETWEEN SQUAMOUS EPITHELIUM AND STROMA

\begin{tabular}{|c||c|c|c|c|c|c|c|c|}
\hline \multirow{2}{*}{$\begin{array}{c}\text { Base block } \\
\text { size }\end{array}$} & \multicolumn{7}{|c|}{ Accuracy (\%) when base blocks are down-sampled from } \\
\cline { 2 - 10 } & $40 \mathrm{X}$ & $20 \mathrm{X}$ & $10 \mathrm{X}$ & $8 \mathrm{X}$ & $5 \mathrm{X}$ & $4 \mathrm{X}$ & $2 \mathrm{X}$ & $1 \mathrm{X}$ \\
\hline \hline $500 \times 500$ & 91.0 & 91.5 & 92.3 & 91.5 & 89.8 & 89.2 & 89.2 & 82.4 \\
\hline $400 \times 400$ & 89.9 & 91.0 & 91.5 & 90.9 & 89.5 & 90.3 & 85.5 & 79.0 \\
\hline $300 \times 300$ & 90.3 & 91.0 & 89.7 & 88.1 & 89.8 & 88.1 & 84.1 & $\mathrm{n} / \mathrm{a}$ \\
\hline $250 \times 250$ & 88.2 & 90.0 & 87.5 & 87.5 & 87.0 & 87.6 & 78.9 & $\mathrm{n} / \mathrm{a}$ \\
\hline $200 \times 200$ & 85.9 & 86.5 & 87.1 & 88.8 & 86.3 & 86.1 & 79.3 & $\mathrm{n} / \mathrm{a}$ \\
\hline $150 \times 150$ & 86.3 & 85.6 & 85.0 & 85.7 & 82.8 & 81.8 & $\mathrm{n} / \mathrm{a}$ & $\mathrm{n} / \mathrm{a}$ \\
\hline $125 \times 125$ & 84.3 & 84.4 & 84.4 & 85.1 & 83.0 & 79.5 & $\mathrm{n} / \mathrm{a}$ & $\mathrm{n} / \mathrm{a}$ \\
\hline $100 \times 100$ & 80.7 & 81.8 & 81.7 & 80.1 & 79.4 & $\mathrm{n} / \mathrm{a}$ & $\mathrm{n} / \mathrm{a}$ & $\mathrm{n} / \mathrm{a}$ \\
\hline $62 \times 62$ & 77.2 & 77.5 & 77.0 & $\mathrm{n} / \mathrm{a}$ & $\mathrm{n} / \mathrm{a}$ & $\mathrm{n} / \mathrm{a}$ & $\mathrm{n} / \mathrm{a}$ & $\mathrm{n} / \mathrm{a}$ \\
\hline $50 \times 50$ & 73.1 & 75.2 & 72.9 & $\mathrm{n} / \mathrm{a}$ & $\mathrm{n} / \mathrm{a}$ & $\mathrm{n} / \mathrm{a}$ & $\mathrm{n} / \mathrm{a}$ & $\mathrm{n} / \mathrm{a}$ \\
\hline $25 \times 25$ & 69.1 & 67.0 & $\mathrm{n} / \mathrm{a}$ & $\mathrm{n} / \mathrm{a}$ & $\mathrm{n} / \mathrm{a}$ & $\mathrm{n} / \mathrm{a}$ & $\mathrm{n} / \mathrm{a}$ & $\mathrm{n} / \mathrm{a}$ \\
\hline
\end{tabular}

\section{E. Image Size and Resolution}

To reduce processing time and memory requirements, it was decided to perform a coarse block-based segmentation at a lower resolution, followed by a fine tuning of boundary blocks (subdividing the coarse blocks into four subblocks, and reclassifying at a higher resolution). These image blocks need to be large enough to include the features necessary for the coarse segmentation, but small enough to give a meaningful boundary. The question becomes one of choosing the best combination of image resolution and block size.

To determine the best block sizes and resolutions, a grid of 88 image resolutions and block sizes is built. Block sizes smaller than $10 \times 10$ pixels are not considered.

For each iteration of the grid-search for image size and resolution, 1000 blocks from each combination are selected. Texture feature vectors for the 1000 blocks are calculated and then classified using SVM and a fivefold cross-validation.

The classification between squamous epithelium and stroma is the most important and difficult one. Therefore, grid-search is firstly performed between these two categories. Results are shown in Table I. When the $500 \times 500$ base blocks at $40 \mathrm{X}$ magnification are down-sampled to give $25 \times 25$ pixels at $2 \mathrm{X}$ magnification, classification still achieves $89.2 \%$ accuracy. So for coarse segmentation, we choose $500 \times 500$ blocks at $2 \mathrm{X}$ magnification (noticing the drop-off at $1 \mathrm{X}$ ). For boundary fine tuning, we notice that (the fourth row) blocks of size $250 \times 250$ achieve good accuracy (maximum $90.0 \%$ ) at $20 \mathrm{X}$.

The grid-search processes for the other three binary classifications follow a similar process (see below).

\section{F. Segmentation of Squamous Epithelium}

After the grid-search for the best block sizes and resolutions for the four binary classification tasks, we are able to use the following eight steps for the segmentation of squamous epithelium from digital slides. Each step is explained below. In order to illustrate the eight-step segmentation process, a small digital slide (Fig. 2(a)) is used as an example. This digital slide is $23000 \times 20000$ pixels at $40 \mathrm{X}$ magnification, which is roughly the glass slide region of $5.4 \mathrm{~mm} \times 4.6 \mathrm{~mm}$.

1) Step 1: Image Partitioning: Scanned digital slides are partitioned into $500 \times 500$ pixel blocks at $40 \mathrm{X}$ magnification, and stored in JPEG format.

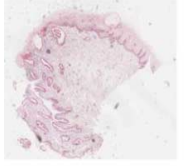

(a)

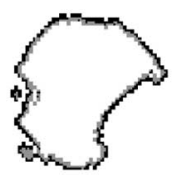

(d)

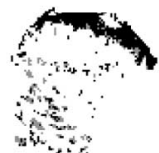

(g)

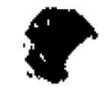

(b)

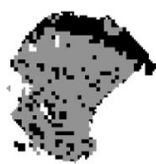

(e)

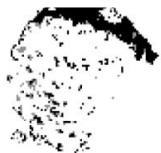

(h)

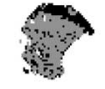

(c)

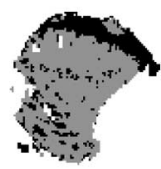

(f)

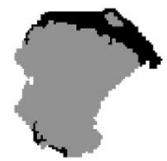

(i)
Fig. 2. Illustration of the eight-step segmentation process. (a) Original digital slide of $23000 \times 20000$ pixels. (b) Result from step 2 (tissue is black). (c) Result from step 3 (squamous epithelium is black and stroma is gray). (d) Reclassified 2-block thick boundary from step 4 (black is the newly generated background). (e) Boundary refined result from step 4 and 5. (f) Boundary expanding-shrinking from step 5. (g) Result from step 6 (columnar epithelium is gray). (h) Result from step 7 (RBCs are gray). (i) Final result from step 8.

2) Step 2: Tissue versus Background at 2X: Digital slides are classified as either background or tissue. First, the $500 \times 500$ pixel blocks at $40 \mathrm{X}$ are converted to gray scale, and then downsampled to give $25 \times 25$ pixel blocks at $2 \mathrm{X}$. The eight texture features for each $2 \mathrm{X}$ block are then calculated, and classified using SVM. An example is shown in Fig. 2(b).

3) Step 3: Squamous Epithelium versus Stroma at 2X: All 14 texture features for each $2 X$ tissue block are calculated. The eight texture features already calculated in step 2 are reused. Then, the tissue block is classified. An example is shown in Fig. 2(c).

4) Step 4: Tissue versus Background Boundaries at 20X: Blocks at the boundary between background and tissue (the borders inside and outside the tissue region) are reclassified.

A boundary $500 \times 500$ pixel block at $40 \mathrm{X}$ is firstly partitioned into four $250 \times 250$ pixel blocks. These four blocks are then down-sampled to give four $125 \times 125$ pixel blocks at $20 \mathrm{X}$. As in step 2, eight texture features are used, and the four subblocks are reclassified to be either tissue or background. An example is shown in Fig. 2(d).

5) Step 5: Squamous Epithelium Versus Stroma at 20X: Step 5 consists of two tasks. First, the boundary tissue blocks between squamous epithelium and stroma are subdivided into four, and classified to be either squamous epithelium or stroma, as in step 4. Secondly, a boundary expanding-shrinking process is used to refine the boundaries between squamous epithelium and stroma. An example of the result of these two tasks is shown in Fig. 2(e) and (f).

6) Step 6: Squamous Epithelium versus Columnar Epithelium at $8 X$ : The segmented squamous epithelium from step 5 is further processed. $250 \times 250$ pixel squamous epithelium blocks at $40 \mathrm{X}$ are down-sampled to be $50 \times 50$ pixels at $8 \mathrm{X}$ magnification. As before, they are classified to be either columnar epithelium or squamous epithelium [Fig. 2(g)]. 
7) Step 7: Squamous Epithelium versus RBCs at 10X: Similar to step 6, the segmented squamous epithelium from step 5 are processed to identify RBCs. Blocks at $40 \mathrm{X}$ are downsampled to be $62 \times 62$ pixels at $10 \mathrm{X}$. An example is shown in Fig. 2(h).

8) Step 8: Remove Misclassifications: The texture-based segmentation can occasionally give erroneous results because of misclassifications. Some of these "rogue" blocks can be identified and reclassified using medical pathology rules, such as the following.

- Isolated tissue blocks surrounded by background are converted to background.

- Tissue-surrounded isolated background blocks are converted to tissue.

- Small regions of squamous epithelium surrounded by background/nonsquamous epithelium tissue are replaced by their surrounding material.

- Small regions of nonsquamous epithelium tissue surrounded by background/squamous epithelium are replaced by their surrounding material.

The application of these rules is illustrated in Fig. 2(i).

\section{G. Results and Analysis}

1) Ground Truth Image Data: For measuring the robustness of segmentation, ground truth images $(G T)$ are acquired from manual segmentation, where boundary blocks are also regarded as squamous epithelium. Under the supervision of pathologists, manual segmentation is performed by drawing regions using Aperio's ImageScope software.

In this application, we choose the following three measurements to indicate how good the proposed segmentation method is.

- Accuracy (Acc): how many blocks correctly classified as either squamous epithelium or nonsquamous epithelium.

- False Positive Proportion $(F P)$ : the proportion (\%) of nonsquamous epithelium blocks wrongly classified as squamous epithelium.

- False Negative Proportion $(F N)$ : the proportion (\%) of squamous epithelium blocks wrongly classified as nonsquamous epithelium.

2) Results: Each of the images is segmented automatically using the proposed method, and the resulting block classifications are compared with the ground truth segmentations. Tests are performed firstly using the 20 "seen" digital slides, which were used for the training of the support vectors. Results for these 20 slides are shown in Table II (slides J3 and J19 were not included as their segmentation was not supervised by pathologists). Then, the tests are performed on the 11 "unseen" images. Results are shown in Table III. Results for digital slide $J 15$ is illustrated in Fig. 3.

As expected, results for the 11 unseen test cases are slightly poorer than the results from the 20 known cases, with the average accuracy dropping about $2 \%$. The reason for the decrease of accuracy is that the 11 test slides were not used in the training of the support vectors. Therefore, SVM has not encountered the texture feature differences between training and testing data, which in turn affects the performance. This problem can be improved by building up a larger training set.

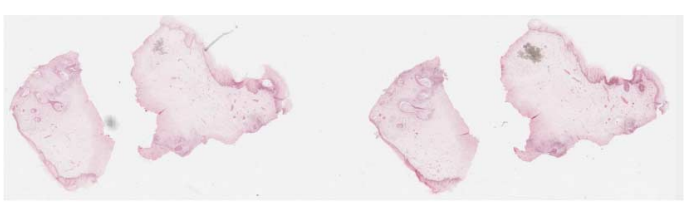

(a)
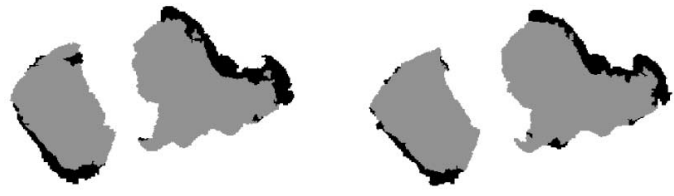

(b)
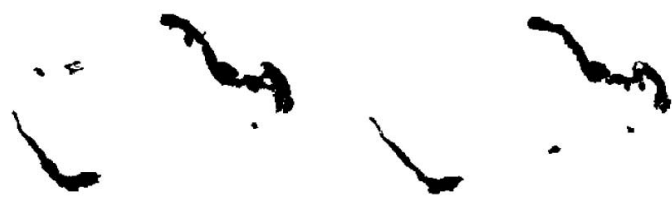

(c)

Fig. 3. Segmentation result of digital slide J15: (a) J15 of the size $152000 \times 41500$ pixels, (b) system segmentation result, (c) GT (black regions are squamous epithelium and white are nonsquamous epithelium.).

TABLE II

Segmentation Results FOR THE 20 "SEeN" Digital Slides

\begin{tabular}{|c||c|c|c||c|c|c|c|}
\hline Name & $A c c$ & $F P$ & $F N$ & Name & $A c c$ & $F P$ & $F N$ \\
\hline \hline J01 & 98.21 & 0.98 & 0.81 & J13 & 95.10 & 3.67 & 1.23 \\
\hline J02 & 97.16 & 0.90 & 1.94 & J14 & 96.41 & 1.28 & 2.31 \\
\hline J04 & 92.84 & 1.39 & 5.77 & J15 & 98.69 & 0.53 & 0.79 \\
\hline J05 & 95.05 & 3.73 & 1.22 & J16 & 97.58 & 1.20 & 1.22 \\
\hline J06 & 97.61 & 1.73 & 0.66 & J17 & 97.92 & 1.16 & 0.92 \\
\hline J07 & 90.32 & 9.13 & 0.55 & J18 & 97.94 & 0.66 & 1.40 \\
\hline J08 & 96.86 & 1.13 & 2.01 & J20 & 97.56 & 1.61 & 0.83 \\
\hline J09 & 95.30 & 0.91 & 3.79 & J21 & 96.72 & 0.29 & 2.99 \\
\hline J10 & 94.50 & 3.18 & 2.32 & J22 & 96.69 & 2.24 & 1.07 \\
\hline J11 & 96.25 & 0.79 & 2.96 & Ave. & 96.23 & 1.92 & 1.86 \\
\hline J12 & 95.83 & 1.83 & 2.33 & STD & 0.02 & 0.02 & 0.01 \\
\hline
\end{tabular}

TABLE III

SEgmentation Results For the 11 "UnSEen” Digital SLides

\begin{tabular}{|c||c|c|c||c|c|c|c|}
\hline Name & $A c c$ & $F P$ & $F N$ & Name & $A c c$ & $F P$ & $F N$ \\
\hline \hline C01 & 95.15 & 3.90 & 0.96 & C07 & 96.98 & 1.61 & 1.41 \\
\hline C02 & 96.45 & 1.88 & 1.67 & C08 & 95.41 & 2.89 & 1.70 \\
\hline C03 & 94.55 & 3.97 & 1.48 & C09 & 92.29 & 5.99 & 1.73 \\
\hline C04 & 96.90 & 1.96 & 1.14 & C10 & 95.28 & 4.44 & 0.27 \\
\hline C05 & 98.00 & 1.50 & 0.50 & C11 & 97.91 & 0.71 & 1.38 \\
\hline C06 & 77.81 & 21.17 & 1.02 & Ave. & 94.25 & 4.25 & 1.21 \\
\hline \multicolumn{1}{|r|}{} & & & & STD & 0.06 & 0.06 & 0.01 \\
\hline
\end{tabular}

3) Execution Speed: The segmentation system is currently implemented in Matlab, and running on a PC with a dual-core Pentium $43.40 \mathrm{GHz}$ processor and $2 \mathrm{~GB}$ RAM. The execution of the entire segmentation process is time consuming. In the test of a typical digital slide (Fig. 3(a) $152000 \times 41500$ pixels), the entire segmentation process takes approx. $2 \mathrm{~h} 52 \mathrm{~min}$. The image partitioning process is the bottleneck and takes $63 \%$ of the entire processing time. There is considerable scope for optimization of this phase. 


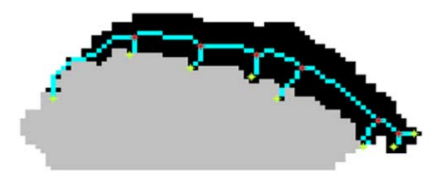

(a)

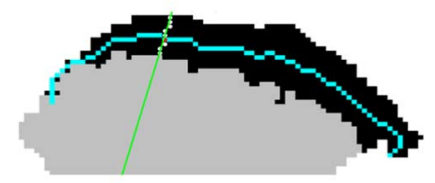

(c)

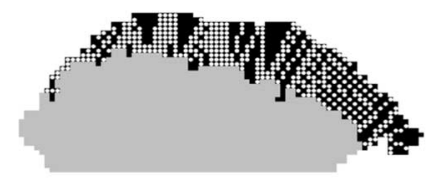

(e)

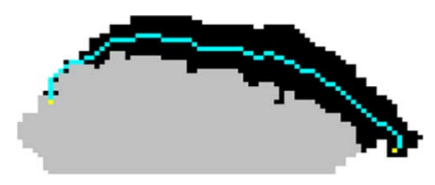

(b)

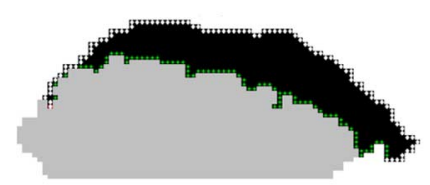

(d)

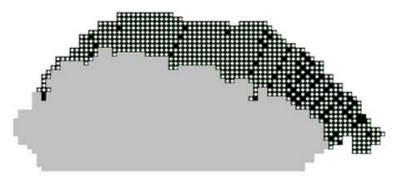

(f)
Fig. 4. (a) Skeleton of squamous epithelium. (b)Main axis of the skeleton. (c) Perpendicular line function. (d) Top and bottom boundaries of squamous epithelium. (e) Sectors and isolated squamous epithelium points not belonging to any perpendicular lines. (f) Improved perpendicular lines.

\section{Measurements of Squamous EPITHElium AND DIAGNOSIS OF CIN}

The diagnosis of CIN is based on measurements from the segmented squamous epithelium, using manual classification by pathologists as "ground truth" training data. Pathologists were observed to return their results in terms of regions of squamous epithelium, giving a diagnosis for each region (i.e., Normal, CIN I, CIN II or CIN III). Our approach aims to emulate this. First, small lines of blocks through the squamous epithelium (perpendicular to its medial axis) are first identified and classified into one of the above four diagnostic categories. Then, regions are formed by combining neighbouring lines into regions based on individual diagnostic outcome, and an overall regional diagnosis is produced.

\section{A. Perpendicular Lines}

A perpendicular line is a line of blocks passing through the squamous epithelium, perpendicular to its medial axis. The blocks are $250 \times 250$ pixel blocks at $40 \mathrm{X}$ magnification. The line traces an 8-connected path through the squamous epithelium. The two ends of the line intersect with the top and bottom boundary of squamous epithelium.

Pathologists diagnose the presence and different stages of CIN by judging the precancerous changes and abnormal cellular growth in the squamous epithelium layer from the bottom (basal) layer to the top (superficial) layer. As the lines follow this direction of progression, the measurement of precancerous changes in squamous epithelium can be modeled by measuring these changes along the lines.

The perpendicular lines are obtained in six steps.

1) Step 1: Skeletonisation of Squamous Epithelium: First, the segmentation result image [see above and Fig. 3(b)] is partitioned into connected regions so that each region contains one piece of continuous squamous epithelium.

For each separate region, skeletonisation using the medial axis transformation is applied [see Fig. 4(a)].
2) Step 2: Main Axis Identification: The resulting skeleton can have many branches which need to be trimmed off. Experiments show that the longest path reflects the major axis. An example is in Fig. 4(b).

3) Step 3: Sequential Path Along the Main Axis: The pixels on the main axis are formed into an ordered sequence, and the start and end points of the axis are identified, based on their connectivity. The start point is distinguished from the end point based on clockwise direction.

4) Step 4: Perpendicular Line Function: A perpendicular line for each point on the main axis is defined. Given a point $\left(X_{i}, Y_{i}\right)$ on the axis, the slope of the axis at that point is calculated based on its four neighbouring pixels along the axis. Two pixels on either side are used to smooth variations. The slope of the perpendicular line $\left(a_{i}\right)$ is calculated, and the perpendicular line for each point $\left(X_{i}, Y_{i}\right)$ is defined by

$$
y=a_{i} \times x+\beta_{i}
$$

where

$$
\beta_{i}=Y_{i}-a_{i} \times X_{i}
$$

5) Step 5: Identifying Blocks on Each Perpendicular Line: Using the perpendicular line function above, we can identify a line of $250 \times 250$ blocks of pixels along (i.e., closest to) a perpendicular line. This is done for each point on the axis. An example is shown in Fig. 4(c). The path begins at the "bottom" (basal) boundary of the squamous epithelium, and ends at its intersection with the "top". Normally, the bottom boundary is adjacent to stroma, and is identified by this. Fig. 4(d) illustrates the result.

6) Step 6: Refining Perpendicular Lines: Not all squamous epithelium blocks are covered by perpendicular lines, which leave a number of squamous epithelium blocks, as shown in Fig. 4(e). We call these points uncovered points. To reduce the number of uncovered points, this step focuses on uncovered points on the top and bottom boundaries, and seeks to connect them with additional perpendicular lines through the axis. After this step, the number of uncovered points is not significant (in this example, 72 points and $9.81 \%$ of squamous epithelium). An example is shown in Fig. 4(f).

\section{B. Segmentation of Nuclei}

Some of the pathological diagnostic clues for CIN are: pleomorphism, superficial maturation and loss of polarity [32]. These can be quantitatively assessed by measuring tissue-level and cellular-level changes in squamous epithelium. These changes are all related to the measurements of nuclei, such as individual nuclei morphologies and their inter-relationships. Therefore, for the diagnosis of CIN, the nuclei must be segmented. In this study we use an incremental color-based thresholding method for segmentation after performing pre-processing, namely color normalization and contrast-limited adaptive histogram equalization (CLAHE).

1) Pre-Processing: Pre-processing is necessary to normalize out the color, intensity and contrast differences of digital slides, which can be the result of the staining process and other factors. The color transfer technique proposed in [33] is used to perform 


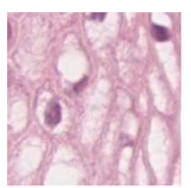

(a)

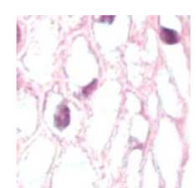

(b)

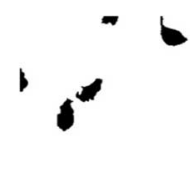

(c)
Fig. 5. Example of nuclei segmentation. (a) Image block. (b) Color normalized (a). (c) Segmented nuclei block.

color normalization. Study [34] suggests the usefulness of it in normalizing H\&E stained histological images. CLAHE [35] is also used to further enhance the contrast of image blocks. Study [36] and experiments show that the use of 20 bins is appropriate.

2) Incremental Thresholding: Incremental thresholding is the technique which segments nuclei from each of the bins of the histogram obtained using CLAHE. All segmented nuclei across all the bins are combined to give the final result, illustrated in Fig. 5.

\section{Perpendicular Line Features}

Cellular-level and tissue-level characteristics measure not only the individual nuclei features, but also inter-relationships among nuclei. Many features are used in the literature [36], [37], such as average nuclei area and features derived from Delaunay triangulation $(D T)$. We choose the following four features for each of the $250 \times 250$ pixel block at $40 \mathrm{X}$ magnification:

- nucSize: average area of nuclei (cellular-level);

- nucDen: number of nuclei in a block (tissue-level);

- mArea: average area of DT (tissue-level);

- mEdge: average edge length of DT (tissue-level).

Tests show that the length of perpendicular lines can range from 1 to more than 20 blocks, depending on the thickness of squamous epithelium. 1-D linear interpolation is used to standardise all perpendicular line feature vectors to a standard length. The interpolated feature vectors have 16 values.

\section{Perpendicular Line Classification}

Perpendicular lines are classified into one of four categories: Normal, CIN I, CIN II and CIN III. Multi-category SVM is chosen to perform this task.

1) Perpendicular Line Feature Database: The 20 digital slides from the first batch (batch-J) are diagnosed and marked by a pathologist (Pathologist A). The other 11 digital slides from the second batch (batch-C) are diagnosed and marked by two pathologists independently (Pathologist A and B). The perpendicular lines are found in all 31 slides. Apart from some regions which are marked as Koilocytosis, the perpendicular lines (26430 lines in total) are taken for training or testing. 8915 lines are selected for training.

If we take the diagnosis made by Pathologist A, for the selected 8915 training cases, the percentage of each diagnostic category is listed in Table IV.

2) Imbalanced Data: Table IV suggests that the number of training cases for each diagnostic category is imbalanced, especially for CIN I and CIN II cases, because the 31 digital slides, although large by current standards, do not cover a sufficiently
TABLE IV

Number of TRAINING CASES FOR EACH DiAgNOSTIC CATEGORY

\begin{tabular}{|c||c|c|}
\hline Normal & 4681 Cases & $52.51 \%$ \\
\hline CIN I & 796 Cases & $8.93 \%$ \\
\hline CIN II & 982 Cases & $11.02 \%$ \\
\hline CIN III & 2456 Cases & $27.55 \%$ \\
\hline Total & 8915 Cases & $100 \%$ \\
\hline
\end{tabular}

wide range of situations. Over-sampling [38] is used to deal with this data imbalance problem. After over-sampling, the data set for the training contains $4681 \times 4=18724$ perpendicular lines.

3) Test Strategies: The chosen test strategies should be able to reflect the following three types of data differences when selecting the SVM training set and testing set. The types of difference are:

I: between lines taken from the same digital slide;

II: between lines taken from different slides, but from the same batch;

III: between lines taken from different slides and batches.

As summarized in [39], popular test strategies are: k-fold cross-validation; the use of separate training and testing sets; and not using a separate evaluation set. In this study we use the latter two test strategies in order to reveal the above mentioned three types of data differences. "Not using a separate evaluation set" means the training and testing data can be from the same digital slide, which could be used to test the Type I difference. Type II and III differences can be tested using separate training and testing sets. If the training and testing sets are from the same batch, Type II difference is tested, whereas if they are taken from different batches, Type III difference can be tested. Therefore, the following test strategies are designed.

- Test Strategy I (J20C11): select a subset of $n$ perpendicular lines from all 31 slides for SVM training. The remaining $(18724-n)$ lines are used for testing. This test strategy reflects Type I Difference.

- Test Strategy II (J13C7): select a subset of $n$ lines from 20 digital slides (13 from batch-J and seven from batch-C) for training. The remaining $(18724-n)$ lines are used for testing. These testing lines are taken from 11 slides (seven from batch-J and four from batch-C) which are not used for SVM training. This strategy reflects Type II difference.

- Test Strategy III (J20): select a subset of $n$ lines only taken from the 20 slides in batch-J. These $n$ lines are used for training. The remaining $(18724-n)$ lines are taken from the 11 digital slides from batch-C and are used for testing. This test strategy reflects Type III Difference.

4) Classification of Perpendicular Lines: Multi-category SVM uses the RBF kernel for this targeted classification task. The LibSVM tool is used. SVM parameters $C$ and $\gamma$ are obtained using grid-search cross-validations for the perpendicular line features for all the three test strategies.

Four pairs of $C$ and $\gamma$ are obtained from the cross-validation for the training of four independent support vectors. Using the cross-validation accuracies $A c c C V_{i}$ from each of the four independent measurements as weights, a perpendicular line is then classified using a combination of the posterior probabilities 


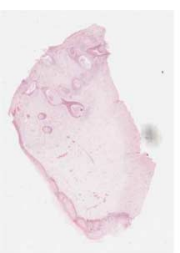

(a)

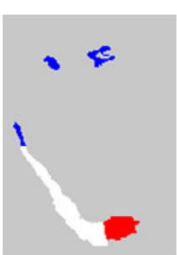

(b)

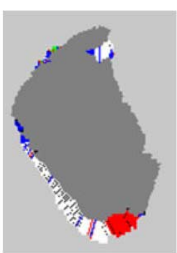

(c)

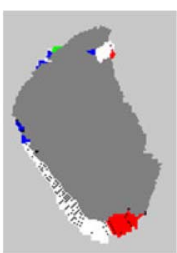

(d)

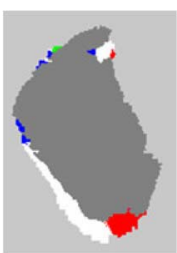

(e)

Fig. 6. Diagnosis of CIN. (a) Original RGB image. (b) Diagnoses marked by a pathologist. (c) Combined perpendicular line diagnoses. (d) Removal of misclassifications. (e) Diagnoses for missed image blocks. (For image (b)-(e), white stands for Normal, red is CIN I, green is CIN II, and blue is CIN III).

$\operatorname{Prob}_{k}(i)$ from SVM predictions and $A c c C V_{i}$. The perpendicular line is then classified to be the category which achieves the highest value in

$$
\sum_{i=1}^{4} \operatorname{Prob}_{k}(i) \bullet A c c C V_{i} .
$$

\section{E. Diagnosis of CIN}

The diagnosis of the various stages of CIN is achieved by combining the perpendicular line classification results, followed by post-processing and visualization.

The post-processing includes three tasks. First, obvious misclassifications of lines are eliminated. Secondly, those pixel blocks at the intersection of differently classified lines are resolved by a simple voting scheme. Finally, uncovered squamous epithelium blocks borrow their nearest neighbors' diagnoses. An example is shown in Fig. 6, where Fig. 6(e) is the visualized final result.

\section{F. Results and Analysis}

This section first evaluates the results of perpendicular line identification and segmentation of nuclei. Then, the four individual perpendicular line features are analyzed, followed by the result and evaluation of perpendicular line classifications. Inter-observer variability is then considered. Finally, the results for the overall diagnosis of CIN is presented and evaluated.

1) Perpendicular Line Identification: The qualitative measurements of how accurate perpendicular lines are identified use visual assessment.

Most perpendicular lines are identified correctly over 31 digital slides. Skeletonisation is clearly effective. In most cases, lines are perpendicular to the top and bottom boundaries. In the test of all 31 digital slides, on average $8.86 \%$ (14.06\% maximum) of the blocks are uncovered.

In some nonstandard tissue configurations, the accuracy of the perpendicular line identification process can depend on the morphology of the segmented squamous epithelium. Usually, wrong identification can arise in the following situations.

- For short, thick squamous epithelium regions, it is not clear where the main axis should be, so the orientation of the longest branch skeleton is sometimes perpendicular to the correct direction.

- Some regions of squamous epithelium can be detached from stroma. All neighboring pixels around the boundary will be background rather than stroma.

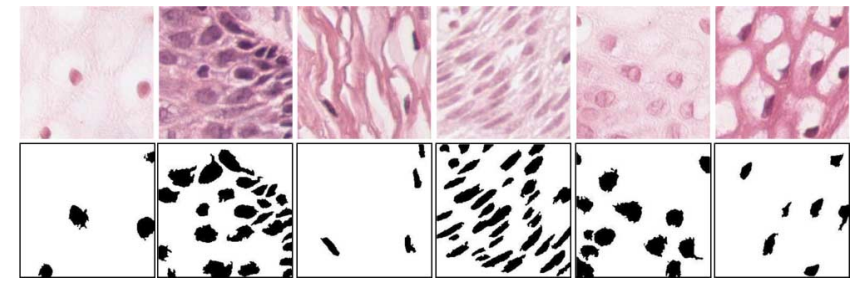

Fig. 7. Six examples of segmented nuclei.

TABLE V

ACCURACIES For INDIVIDUAL PERPENDICULAR LiNE FEATURES

\begin{tabular}{|c|c|c|c|c|c|c|}
\hline & \multirow{2}{*}{$\begin{array}{c}\text { Training } \\
\text { Sets } \\
\text { Sizes } \\
\end{array}$} & \multirow{2}{*}{$\begin{array}{c}\text { Testing } \\
\text { Sets } \\
\text { Sizes } \\
\end{array}$} & \multicolumn{4}{|c|}{ Individual Line Features (\%) } \\
\hline & & & nucSize & nucDen & mArea & mEdge \\
\hline I & 93 & 9362 & & & & 1 \\
\hline II & 5172 & 5172 & 94 & 91.42 & 62.51 & 82.08 \\
\hline III & 7576 & 7576 & 97.19 & 93.48 & 89.57 & 90.47 \\
\hline
\end{tabular}

TABLE VI

Perpendicular Line Classification Results for Three Test Strategies

\begin{tabular}{|c||c||c|c||c|c|}
\hline \multirow{2}{*}{ Test Strategies } & \multirow{2}{*}{ I } & \multicolumn{2}{c||}{ II } & \multicolumn{2}{|c|}{ III } \\
\cline { 3 - 6 } & & Test A & Test B & Test A & Test B \\
\hline \hline Test Samples & 9362 & 5172 & 6657 & 7576 & 7327 \\
\hline \hline Accuracy & $96.5 \%$ & $93.3 \%$ & $65.4 \%$ & $98.4 \%$ & $52.1 \%$ \\
\hline Unweighted Kappa & 0.9536 & 0.9108 & 0.3827 & 0.9787 & 0.3175 \\
\hline Quadratic Kappa & 0.9308 & 0.9072 & 0.6010 & 0.9618 & 0.5178 \\
\hline
\end{tabular}

TABLE VII

INTER-OBSERVER VARIABILITY OVER 11 SLIDES FROM 2ND BATCH (BATCH-C)

\begin{tabular}{|c|c|c|c|c|c|c|}
\hline \multicolumn{2}{|c|}{} & \multicolumn{4}{|c|}{ A } & Unweighted \\
\cline { 3 - 6 } & Normal & CIN I & CIN II & CIN III & Kappa \\
\hline \multirow{4}{*}{ B } & Normal & $\mathbf{2 9 3 6}$ & 0 & 0 & 0 & 0.5213 \\
\cline { 2 - 6 } & CIN I & 1416 & 0 & 0 & 0 & -0.0975 \\
\cline { 2 - 5 } & CIN II & 812 & 195 & $\mathbf{1 4 1}$ & 0 & -0.0685 \\
\cline { 2 - 5 } & CIN III & 0 & 80 & 1645 & $\mathbf{2 2 9 6}$ & 0.5914 \\
\hline \multicolumn{6}{|c|}{ Overall Unweighted Kappa } & 0.3811 \\
\hline \multicolumn{6}{|c|}{ Overall Quadratic Weighted Kappa } & 0.8034 \\
\hline
\end{tabular}

2) Nuclei Segmentation Results: The accuracy of the nuclei segmentation is measured by comparing automatic segmentation results with manually segmented nuclei. In tests, our nuclei segmentation method identified $86.7 \%-91.2 \%$ of nuclei. Some examples of nuclei segmentation results are shown in Fig. 7.

3) Individual Perpendicular Line Features: In the test of individual perpendicular line features, Pathologist A's diagnoses over all 31 slides are used as ground truth. The system's classifications are compared with these. Results are shown in Table V.

Results show that, except for mArea in Test Strategy II, $>80 \%$ accuracy is achieved. These findings show that the four 
TABLE VIII

Results for the Diagnosis of CIN Over EntiRe Digital SLides Using ThreE Test STRATEgies

\begin{tabular}{|c|c|c|c|c|c|c|c|c|c|}
\hline \multirow{3}{*}{ Measurements } & \multirow{2}{*}{\multicolumn{2}{|c|}{ Strategy I }} & \multicolumn{4}{|c|}{ Strategy II } & \multicolumn{3}{|c|}{ Strategy III } \\
\hline & & & \multicolumn{2}{|c|}{ Test A } & \multicolumn{2}{|c|}{ Test B } & Test A & \multicolumn{2}{|c|}{ Test B } \\
\hline & Path. A & Path. B & Path. A & Path. B & Path. A & Path. B & Path. A & Path. A & Path. B \\
\hline Accuracy & $94.61 \%$ & $84.48 \%$ & $94.87 \%$ & $83.24 \%$ & $74.90 \%$ & $72.85 \%$ & $95.27 \%$ & $57.06 \%$ & $60.40 \%$ \\
\hline Unweighted Kappa & 0.9032 & 0.7048 & 0.9186 & 0.7114 & 0.3763 & 0.3324 & 0.8961 & 0.3373 & 0.3123 \\
\hline Quadratic Kappa & 0.9141 & 0.8470 & 0.9317 & 0.8363 & 0.5470 & 0.5860 & 0.9012 & 0.4954 & 0.4368 \\
\hline
\end{tabular}

proposed perpendicular line features are valid measurements. For all three test strategies, nucSize is the most accurate feature $(>94 \%)$. These four line features are measuring the pathological diagnostic clues: pleomorphism, superficial maturation and loss of polarity. The assessment of the individual line features confirms the validity and importance of these diagnostic clues.

4) Classification of Perpendicular Lines: To evaluate the four perpendicular line features, accuracy and Cohen's Kappa statistics (both unweighted and quadratic weighted) are used. Cohen's Kappa is a statistical measurement to evaluate the agreement between two observers who classify a number of items into $\mathrm{N}$ mutually exclusive categories. Kappa values greater than 0.75 are considered to represent excellent agreement between observers. Values between 0.40 and 0.75 are regarded as fair to good agreement, and values less than 0.40 are called poor agreement [40].

For all the three test strategies, the perpendicular line features are classified and compared with Pathologist A's manual classification results.

Results (Table VI) show that when testing samples are from the same set of slides as the training set, though using different lines (2nd, 3rd and 5th columns of Table VI), excellent results are obtained, with corresponding accuracies close to $100 \%$, and all Kappa values close to 1 (excellent agreement). When testing lines come from different digital slides but the same batch (fourth column of Table VI), the accuracy value drops to $65.39 \%$, unweighted Kappa becomes poor agreement (0.3827) and quadratic weighted Kappa becomes fair to good agreement. Finally, when testing perpendicular lines from different batches (last column of Table VI), the accuracy value worsens to be $52.12 \%$, unweighted Kappa get poor agreement (0.3827), and quadratic weighted Kappa gets fair to good agreement.

We conclude that the classification of perpendicular lines is robust if the lines for testing are representative of samples from the same slides and from the same batch as the training data. System performance decreases when the lines for testing are from "unseen" images, even from the same batch, which may have different morphological characteristics. This suggests the key factor is to have sufficient and representative training data which covers as many likely tissue scenarios as possible. This is, in retrospect, not a surprising result, but is a reflection of the practical difficulties of obtaining a large set of ground truth data when using such large and complex images. However, the high accuracies obtained when the training data is representative is encouraging.

5) Inter-Observer Variability: One application of our automatic diagnosis system is to use it as a standard for comparing the diagnoses from different pathologists (inter-observer variability) - or even comparing the performance of the same pathologist at different times (intra-observer variability). We have experimented with the former of these two possibilities.

A previous study [3] measured the inter-observer variability in the diagnosis of CIN. It did two rounds of tests over 100 biopsies with seven experienced pathologists. Results showed poor agreement with unweighted Kappa of 0.354 and 0.357 , and excellent quadratic weighted Kappa of 0.772 and 0.778 . When looking at individual unweighted Kappa, except for CIN III which achieved fair to good and excellent agreement (0.523-0. 797), all the other cases (Normal, CIN I, and CIN II) produced poor agreement.

The 11 digital slides from batch- $\mathrm{C}$ were diagnosed and marked by the two pathologists separately. Their inter-observer variability for perpendicular lines is measured, with results shown in Table VII. The figures in the diagonal line of the table show complete agreement between the two pathologists for 5373 perpendicular lines $(56.43 \%)$. When considering Kappa for each diagnostic group, Normal and CIN III cases achieve fair to good unweighted Kappa values, with Kappa values of 0.5213 and 0.5914 . CIN I and CIN II cases get poor agreement $(-0.0975$ and -0.0685$)$.

6) Diagnosis of CIN: The robustness of the diagnosis of CIN is measured using accuracy, unweighted Kappa and quadratic weighted Kappa. For all three test strategies, system diagnoses are compared with both pathologists' diagnoses, where available. Results are shown in Table VIII.

For each test strategy, results obtained from comparing with Pathologist A's diagnoses are better than comparing with Pathologist B. This is because the training of support vectors take Pathologist A's diagnoses as ground truth.

When comparing the inter-observer variability from the results in the second, fourth, sixth, eighth, and ninth columns in Table VIII with [3], the system diagnoses are likely to be better in term of overall unweighted Kappa. A digital slide can have many different regional diagnoses. Therefore, corresponding Kappa values of regional diagnoses for [3] can be a lot lower than the Kappa values for entire digital slides. Study [3] reports the overall unweighted Kappa to be 0.354 and 0.357 measuring entire digital slides, whereas the smallest overall unweighted Kappa in Table VIII is 0.3373 measuring regional diagnosis.

When comparing the inter-observer variability from the results in the second, fourth, sixth, eighth, and ninth columns in Table VIII with Table VII, overall unweighted Kappa all achieve poor agreement $(0.3811$ and 0.3373$)$. The overall quadratic Kappa value in Table VII (excellent agreement, 0.8034 ) is better than its corresponding entry in Table VIII (fair to good agreement, 0.4954). However, with more slides from different batches for training, the system diagnosis might have the potential to address the problem of inter-observer variability. 


\section{CONCLUSION}

The traditional way of diagnosing CIN is subjective, resulting in great inter/intra-observer variability and poor reproducibility. With the use of high-resolution slide scanning devices, ultralarge complex cervical histological slides can be presented in digital form. In this project, the feasibility and process of designing and developing a computer assisted system for the diagnosis of CIN using histological digital slides is investigated.

This paper has described the first reported major study into the automatic classification of CIN, and the first reported work in the segmentation of ROIs from complex histological scenes using ultra-large digital slides. A novel method for the diagnosis of CIN was developed. This method is based on the classification of line features to classify progression of CIN characteristics through squamous epithelium.

This is also the first reported work to quantitatively measure linguistic diagnostic clues of CIN. Correlations between linguistic diagnostic clues of $\mathrm{CIN}$ and individual line features were built and quantitatively measured.

Evaluation of the perpendicular line feature proves that it is robust in classifying lines for the diagnosis of CIN. The robustness of the system's diagnosis is comparable with pathologists when comparing inter-observer variability between the system and pathologists, and among pathologists.

Given sufficient training data, from a suitable set of representative digital slides, the proposed diagnostic system could be the basis of a clinical tool for the assisted diagnosis of CIN, and also as a training tool.

More manual diagnoses by several experts, both in quantity and in reliability, are needed to enhance the robustness of the proposed assisted diagnostic system. The potential of the system to address the problems of inter-observer variability among pathologists is particularly promising.

The processing of digital slides is very time consuming and currently too slow to be used as a clinical diagnostic device. High performance computing techniques are needed (and are being developed) to speed up the system.

\section{REFERENCES}

[1] J. Ferlay et al., "GLOBOCAN 2000: Cancer Incidence, Mortality and Prevalence Worldwide, version 1.0," in IARC Cancer Base No. 5. Lyon, France: Int. Agency for Res. on Cancer Press, 2001.

[2] W. G. McCluggage et al., "Inter- and intra-observer variation in the histopathological reporting of cervical squamous in traepithelial lesion susing a modified bethesda grading system," BJOG: An Int. J. Obstet. and Gynecol., vol. 105, no. 2, pp. 206-210, 1998.

[3] S. M. Ismail et al., "Reporting cervical intra-epithelial neoplasia (CIN): Intra- and interpathologist variation and factors associated with disagreement," Histopathology, vol. 16, no. 4, pp. 371-376, 1990.

[4] C. Molloy et al., "Evaluation of colposcopically directed cervical biopsies yielding a histologic diagnosis of CIN 1,2," J. Lower Genital Tract Dis., vol. 6, no. 2, pp. 80-83, 2002.

[5] D. Soenksen, "The road to digital pathology," in Proc. Pathology Vision, Aperio Digital Pathology Solution Conf., Leeds, U.K., 2006.

[6] D. Soenksen, "The future of digital pathology," in Proc. Pathology Vision, Aperio ScanScope User Conf., Switzerland, 2005.

[7] Digital slides and third party data interchange. Bristol, U.K.: Aperio Technologies, I, 2006.

[8] B. Molnar et al., "Digital slide and virtual microscopy based routine and telepathology evaluation of routine gastrointestinal biopsy specimens," J. Clin. Pathol., vol. 56, no. 6, pp. 433-438, 2003.
[9] J. Burthem et al., "The use of digital 'virtual slides' in the quality assessment of haematological morphology: Results of a pilot exercise involving U.K. NEQAS(H) participants," Brit. J. Haematol. 2005, vol. 130, no. 2, pp. 293-296.

[10] T. Harris et al., "Comparison of a virtual microscope laboratory to a regular microscope laboratory for teaching histology," Anatom. Rec., vol. 265 , no. 1 , pp. 10-14, 2001.

[11] R. K. Kumar et al., "Virtual microscopy for learning and assessment in pathology," J. Pathol., vol. 204, no. 5, pp. 613-618, 2004.

[12] H. Helin et al., "Web-based virtual microscopy in teaching and standardizing gleason grading," Hum. Pathol., vol. 36, no. 4, pp. 381-381, 2005.

[13] M. Lundin et al., "A digital atlas of breast histopathology: An application of web based virtual microscopy," J. Clin. Pathol., vol. 57, pp. 1288-1291, 2004.

[14] R. S. Weinstein et al., "Telepathology overview: From concept to implementation," Hum. Pathol., vol. 32, no. 12, pp. 1283-1283, 2001.

[15] S. S. Costello et al., "Development and evaluation of the virtual pathology slide: A new tool in telepathology," J. Med. Internet Res., vol. 5, no. 2, pp. e11-e11, 2003.

[16] K. Kayser et al., "Towards an automated virtual slide screening: Theoretical considerations and practical experiences of automated tissue-based virtual diagnosis to be implemented in the internet," Diagn. Pathol., vol. 1, pp. 10-10, 2006.

[17] K. A. DiVito and R. L. Camp, "Tissue microarrays-automated analysis and future directions," Breast Cancer Online, vol. 8, 2005.

[18] D. G. Soenksen, Automated Microscopic Inspection of Tissue Microarrays Using Virtual Microscopy. Bristol, U.K.: Aperio Technologies, Inc., 2003.

[19] L. Ficsór et al., "Automated histological classification of colon biopsy samples using image analysis on digital slides," in Proc. 47th Annu. Meeting of the Hungarian Society of Gastroenterology, 2005.

[20] B. M. G. Begelman, E. Rivlin, and E. Sabo, "System for computeraided multiresolution microscopic pathology diagnostics," in System for Computer-Aided Multiresolution Microscopic Pathology Diagnostics. New York: IEEE Computer Society, 2006.

[21] R. Gonzalez and R. Woods, Digital Image Processing, 2nd ed. Englewood Cliffs, NJ: Prentice-Hall, 2002.

[22] S. Arivazhagan and L. Ganesan, "Texture segmentation using wavelet transform," Patt. Recognit. Lett., vol. 24, pp. 1513-1521, 2003.

[23] R. Kamalov et al., "A java application for tissue section image analysis," Comput. Meth. Progr. Biomed., vol. 77, no. 2, pp. 99-99, 2005.

[24] J. Diamond et al., "The use of morphological characteristics and texture analysis in the identification of tissue composition in prostatic neoplasia," Hum. Pathol., vol. 35, no. 9, pp. 1121-1131, 2004.

[25] A. K. Bachoo and J. R. Tapamo, "Texture analysis and unsupervised clustering for segmenting iris images," Prasa, 2005.

[26] K. I. Chang, K. W. Bowyer, and M. Sivagurunath, "Evaluation of texture segmentation algorithms," in Proc. IEEE Computer Vision and Pattern Recognition (CVPR'99), 1999.

[27] A. N. Esgiar et al., "Microscopic image analysis for quantitative measurement and feature identification of normal and cancerous colonic mucosa," IEEE Trans. Inform. Technol. Biomed., vol. 2, no. 3, pp. 197-203, Sep. 1998.

[28] P. Hamilton et al., "Automated location of dysplastic fields in colorectal histology using image texture analysis," J. Pathol., vol. 182, no. 1, pp. 68-75, 1997.

[29] M. S. Lew, Principles of Visual Information Retrieval. New York: Springer, 2001, pp. 51-86.

[30] B. E. Boser, I. M. Guyon, and V. N. Vapnik, "A training algorithm for optimal margin classifiers," in Proc. 5th Annu. Workshop on Computational Learning Theory, Pittsburgh, PA, 1992.

[31] C. W. Hsu, C. C. Chang, and C. J. Lin, A Practical Guide to Support Vector Classification. Taipei, Taiwan, R.O.C.: Dept. Comput. Sci. Inform. Eng., Nat. Taiwan Univ., 2003.

[32] G. J. Price et al., "Computerized diagnostic decision support system for the classification of preinvasive cervical squamous lesions," Hum. Pathol., vol. 34, no. 11, pp. 1193-1193, 2003.

[33] E. Reinhard et al., "Color transfer between images," in Computer Graphics and Applications. New York: IEEE Press, 2001, vol. 21, pp. 34-34.

[34] Y. Y. Wang et al., "A color-based approach for automated segmentation in tumor tissue classification," in Proc. 29th Annu. Conf. IEEE Engineering in Medicine and Biology Society 2007 (EMBS 2007), 2007.

[35] S. M. Pizer et al., "Adaptive histogram equalization and its variations," Comput. Vision Graph. Image Process., vol. 39, no. 3, pp. 355-368, 1987. 
[36] S. J. Keenan et al., "An automated machine vision system for the histological grading of cervical intraepithelial neoplasia (CIN)," J. Pathol., vol. 192 , no. 3, pp. 351-362, 2000.

[37] R. Kamalov et al., "A java application for tissue section image analysis," Comput. Meth. Progr. Biomed., vol. 77, no. 2, pp. 99-99, 2005.

[38] M. W. Gary, "Mining with rarity: A unifying framework," SIGKDD Explor. Newslett., vol. 6, no. 1, pp. 7-19, 2004

[39] C. Demir and B. Yener, Automated Cancer Diagnosis Based on Histopathological Images: A Systematic Survey Rensselaer Polytechnic Inst., Troy, NY, 2005, Tech. Rep. TR-05-09.

[40] J. Fleiss, Statistical Methods for Rates and Proportions, 2 ed. New York: Wiley, 1981.

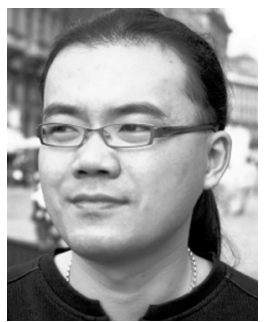

Yinhai Wang received the B.Eng. degree in computer software from Jinan University, Guangzhou, China, in 2001, the M.Sc. degree in software engineering from Napier University, Edinburgh, U.K., in 2002, and the Ph.D. degree in electronics, electrical engineering, and computer science from Queen's University, Belfast, U.K., in 2008.

$\mathrm{He}$ is currently a Software Engineer with VCA Technology Ltd, London, U.K.

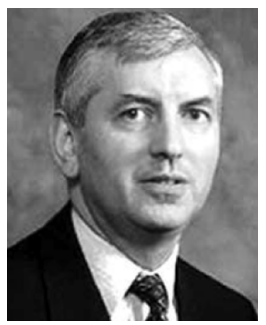

Danny Crookes (M'98) received the B.Sc. degree (first-class) in mathematics and computer science in 1977 and the Ph.D. degree in computer science in 1980, both from Queen's University, Belfast, U.K.

$\mathrm{He}$ is a Professor of Computer Engineering at Queen's University. His current research interests include image processing applications, such as medical imaging and shoeprint recognition, and high-performance image and video processing. He has presented tutorials on parallel image processing at several international conferences, and has some 180 scientific papers in journals and international conferences.

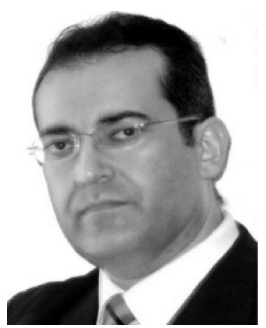

Osama Sharaf Eldin received the M.B.B.Ch. degree in medicine in 1992 and the M.Sc. degree in pathology in 1999, both from Mansura University. $\mathrm{He}$ received the Ph.D. degree in molecular pathology from Queen's University, Belfast, U.K., in 2006.

$\mathrm{He}$ is currently a Special Lecturer in Pathology in the School of Medicine and Medical sciences, University College Dublin, Dublin, Ireland. His research interests include whole virtual slide image analysis, morphometric studies, e-learning methods, as well as short interference RNA protein knockdown, apoptosis, and tumour cell resistance.

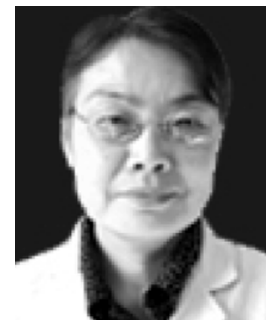

Shilan Wang received the M.B. degree in clinical medicine from Shanxi Medical University, Taiyuan, Shanxi, China, in 1965.

She is currently a Professor of Pathology at Shanxi Modern Woman Hospital, Bosheng Medical Investment Co, Ltd., Taiyuan, Shanxi, China. She has been working in clinical diagnosis since 1970 .

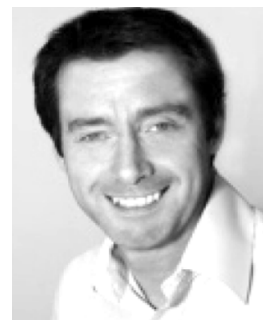

Peter Hamilton received the B.Sc. degree in biology from the University of Ulster, Belfast, U.K., in 1984 , and the Ph.D. degree in quantitative pathology from Queen's University, Belfast, in 1989.

$\mathrm{He}$ is a Professor of Bioimaging and Informatics at Queen's University and a Fellow of the Higher Education Academy. He currently leads research into the use of virtual microscopy, image analysis, and expert systems for training, education, and research in pathology and is the principal developer of the PathXL ${ }^{\mathrm{TM}}$ virtual slide content management system to be used in this project. He has over 100 publications and major research grants in the field of digital pathology.

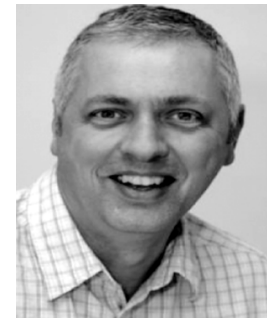

image analysis.
Jim Diamond received B.Sc. degree in engineering in 1983 and the Ph.D. degree in engineering in 1993, both from Queen's University, Belfast, U.K.

$\mathrm{He}$ is a Lecturer of Bio-imaging and Informatics at Queen's University. He currently leads research into the use of virtual microscopy, image analysis, and expert systems for training, education, and research in pathology and is the principal developer of the PathXL ${ }^{\mathrm{TM}}$ virtual slide content management system to be used in this project. He has many peer-reviewed publications in the field of digital pathology and 